\title{
Access to OPC services based on Android platform
}

\author{
Wang Xiao-yan ${ }^{1, a}$, Lu Xuefei, ${ }^{2, b}$ \\ ${ }^{1}$ College of Science, Xi'an Shiyou University, Xi'an, 710065, China \\ ${ }^{2}$ College of Science, Xi'an Shiyou University, Xi'an, 710065, China \\ aemail: 18512262@qq.com, bemail: luxuefei@xsyu.edu.cn.
}

Keywords: OPC; DDE; Android

\begin{abstract}
In order to realize the data access service of Android platform for OPC,Analysis of the OPC of Android, developed a data access service system for PLC based on Android platform, described the system architecture, work flow and communication protocol, Implemented two kinds of state data access of the short connection and the long connection for PLC controller. The system has the advantages of high efficiency, convenient and practical, that engineers can receive data and control system through the android mobile devices whenever and wherever, and make use of existing resources for industrial on-site service.
\end{abstract}

\section{Introduction}

OPC is the abbreviation of Object Linking and Embedding (OLE) for Process Control, it is the application of Microsoft's object linking and embedding technology in process control. OPC is based on OLE/COM/DCOM, adopting the model of Client/Serve, providing a uniform standard for the industrial object-oriented development of automation software, which defines a method of using the Microsoft OS exchange the automation real-time data among clients based on PC.The OPC Server provides many interfaces, the Client can obtain the information of the hardware devices that is connected to OPC Server, without understanding the details of these hardware devices by these interfaces. Program designer can operate different hardware devices by using the same code. Due to the details of network part has been realized by COM/DCOM, it is easy for the Client to remote access to OPC Server simultaneously, which makes the program designer control remote object easier.

The OPC access service on the Android platform is still in the initial stage currently, the corresponding application is relatively rare in the market. Russia developed a supervisory control and data acquisition system -- TeslaSCADA, which is used in the smart phone and android devices. It is the first monitoring system based on OPC UA; The system has high efficiency, convenient and practical. However, TesalSCADA is a paid SDK that won't open to all users. We will develop a new system that can provide PLC data access service on the Android platform.

\section{Architecture design}

The architecture allows the user can access the data of PLC controller on the terminal of the Android platform by means of cross-platform communication.

We take the TCPIPH1 software as OPC Server on Windows platform. The PLC data can be accessed by OPC Server. If the client application wants to access the data of PLC, it needs to obtain from OPC Server. You need to provide interface agent software at both ends platform respectively. Server side agent on Windows platforms can read the data of PLC provided by the OPC Server. After processing the information, Proxy Server side send it to the agent for the Client side. Client side agent and Server side agent located in two different platforms respectively and communicate to each other by wireless Wi-Fi network. Client side agent in Android platform, can obtain the data of the proxy Server, and after processing can provide to the Client application data to meet the needs of users, and users obtained the needed PLC data in the end. 


\section{Android/Windows workflow.}

It begin from the Android client application here, introduces the working process.

First of all, on the Android users, who need to access the PLC data, then write the command that OPC Server can parse on the Client application, that is the command that TCPIPH1 can parse, then send data to the agent for the Client side. Written socket and the length instructions of the command data by proxy Client, then sent to the connected proxy Server side; After the proxy Server receives data, the command that can be parsed is submitted to the OPC Server; By OPC Server access PLC and acquire data that is submitted to the proxy server side; Written socket and the length indication of the data content by proxy Server, then sends the data to the proxy Client of the Android platform; After receiving the data, the proxy client provided it to the client application; Once the client application reads the data, the user can obtain the data content of PLC, realizing PLC controller data access on the Android platform.

\section{Short connection,long connection.}

Short connection and long connection are two kinds of modes when accessing to the data;

Short connection mode is a relatively simple case, the users can convey only one command (for example: users only need a PLC data specified address)to establish short connection mode, the OPC Server access to PLC,only collect a set of data that will be sent to the proxy Server in the supreme level. After that, turn back the data to the proxy client. Finally the Client receives the set of data, short connection end, OPC Server no longer access and collect data.

However, for the users, more often, not only needs a set of data on the PLC, but to update the data on the specified address. In most cases the users can transmit multiple commands, and need multiple sets of data meantime.Therefore, we need to build a more durable, a greater amount of information transmission; continuous data update connection status that is long connection status.

Under the long connection mode, the user can convey multiple orders(for example: the user needs multiple PLC data specified address).Establish long connection mode, the OPC Server access to PLC,collect multiple corresponding sets of data content that will be sent to the proxy Server in the supreme level. After that, turn back the data to the proxy client. Finally the Client receives this set of data. But the connection won't close, these PLC data specified address will be uploaded periodically, and the client will continue to update these data. To implement a new data acquisition, only after the client receive new orders. From the point of time, the duration of the long connection is longer than a short connection.

\section{Two kinds of socket.}

According to the different needs of users, we need to set up short connection and long connection. In order to do that, we also need to provide users with two different kinds of sockets that can set up short connection and long connection in cross-platform communication agent software. Two kinds of sockets is needed for proxy client, to agent two kinds of socket based on windows platform in proxy server.

\section{Communication protocol}

\section{The protocol between the proxy Client/Server.}

In short connection, only single orders can be conveyed, proxy client need to add a head of 32 bit. The length of the command data was written in the head. After receiving the data, the proxy server performs follow-up operations.OPC Server only collect a set of content data, so the proxy Server also need a 32 bit head that indicates the length of the content data. Then the head is passed on to the proxy Client, eventually content is provided to the Client application. Communication is over.

In the long connection, the user can order a single command, can also be multiple orders. When multiple orders, not only need to write the length of data for each order, but the proxy client need to write a new head that indicates the length of all orders. After receiving the data, the proxy server performs follow-up operations.OPC Server collect multiple sets of data, so the proxy Server will write the length of the corresponding content data for each head, at the same time, need to add one more head that indicates the length of all content date. Then the head is passed on to the proxy 
Client, eventually content is provided to the Client application. At the same time, data collection won't stop. The data will be uploading periodically and user data will be updated as a result, until new orders from the Client is received.

\section{Protocol between users and system.}

Due to the user in the Android platform can directly obtain information on the PLC, realizing transparent data transmission. Users give orders to the system according to their own needs. So there is also a corresponding communication protocol between the user and the system. The user can choose to establish long or short connection according to their own data requirement. But from a user's point of view, whether short connection or long connection, there is no different in orders. When users want to convey single orders, all the action needed to do is to input order that can be parsed by TCPIPH1 in command bar, then press the corresponding button of "short/long" connection to send command data. The order is identified for the single command, and the length instructions are written to it. Agent software automatically establishes a connection to get the data, and the returned data is identified as a block that is displayed in the user's data column. When users want to convey multiple orders, all the action needed to do is to input multiple orders that can be parsed by TCPIPH1 in command bar, then press the corresponding button of "short/long" connection to send command data. The orders are identified for the multiple commands, the length instructions and the total length is written to it. Agent software automatically establishes a connection to get the data, and the returned data is identified as multiple blocks that are displayed in the user's data column.

\section{Test results}

On the Android platform, we tested the data access function based on OPC with our own software.

As is shown in figure 1, which is the interface of our own application Android OPC that is developed by you. There are command column and data column, user can type the command in the command bar, and then click the "short connection" or "long connection" button on the bottom to send command. The returned data will be shown in the data column.

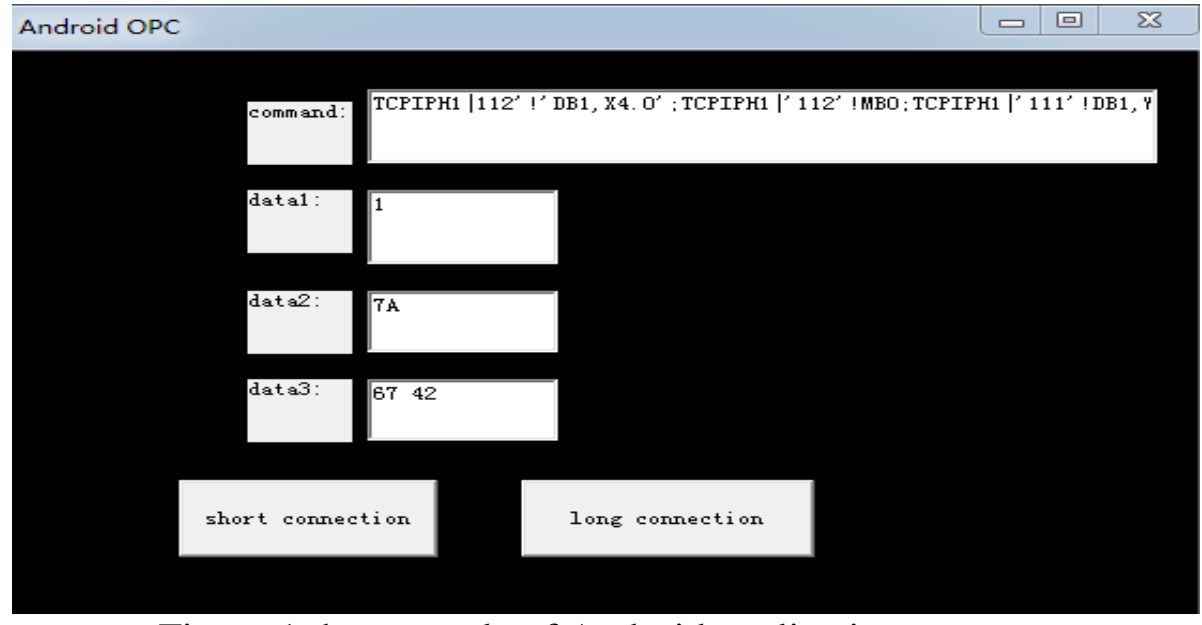

Figure 1 the example of Android application program

In this experiment, we input 3 orders (multiple orders) in the client application, and hope that we will get 3 sets of data from PLC.OPC server is TCPIPH1 on Windows platform, so the input command need to be parsed by TCPIPH1.As is multiple orders, each order is separated by a semicolon. Input orders are as follows:

TCPIPH1|'112'!'DB1,X4.0';TCPIPH1|'112'!mb0;TCPIPH1|'111'!'DB1,w10'

The proxy Server on the Windows platforms will sent the received data to TCPIPH1.after receiving the data, the TCPIPH1 send it back to client. Three groups of data obtained are as follows:

1 


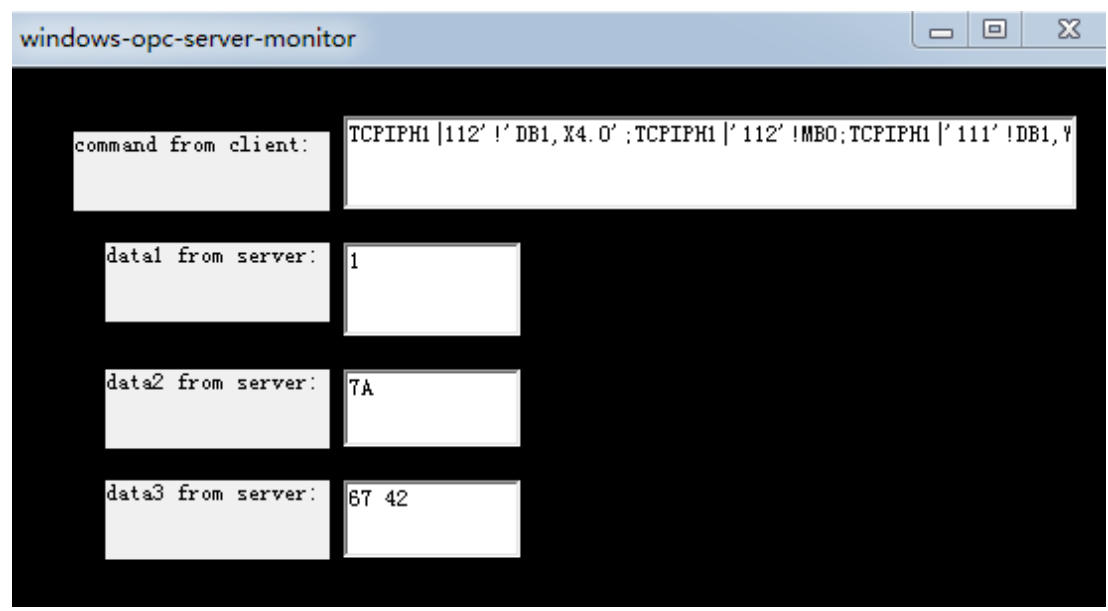

Figure 2 windows agent server

The results from proxy server are shown in figure 2.

Three groups of data on the Android platform can be seen from the figure 2, consistent with the data obtained from Windows platforms. As a result, this system realized the data access from the client application on Android to PLC controller.

\section{Conclusion}

This paper introduces the working process on the Android/Windows, on Android platform, realized data access services based on OPC.completed short and long connection two kinds of state data access service. In the present, with the more powerful mobile devices, and wider application of communication Technologies (wi-fi,3g,4g),the system can achieve the best use of existing resources to service industrial field, Through a Smartphone or other Android mobile devices, let the engineer receive engineering data information and control the system anytime, anywhere, realized OPC cross-platform communication.

\section{References}

[1] Yang Fengsheng.Android Application Development decryption [M].Beijing:Machinery Industry Press,2010

[2] Yu Zhilong etc. Android SDK development examples[M].Beijing:People Post Press,2010

[3] Zeng Jianping etc. Android system architecture and application development research [J]. Microcomputer Information,2011, (09):1-3

[4] Android Distribution of Apps and Cames in Android Market [EB/OK] [2011-05-18]. http://cn.androlib.com/apstatsdownloads.aspx.

[5] Ye Bingfa. Android operating system migration and Key Technologies [D]. Guangzhou:Jinan University,2010:5-10

[6]Zhu Tingting etc. Review of application software based on Android [J]. Computer and telecommunications.2011,(01):42-43

[7] Sun Denghui etc. Development and application of OPC DA client-based .NET [J]. Northern Industrial University,2009,21(3):9-12,18

[8] Han Chao,etc. Android system principle and develop points Comments [M].Beijing: Electronic Industry Press.2010 